\title{
SUBGROUPS LIKE WIELANDT'S IN SOLUBLE GROUPS
}

\author{
CLARA FRANCHI \\ Dipartimento di Matematica pura ed applicata, Università di Padova, Via Belzoni, 7 , \\ I-35131 Padova, Italy
}

(Received 5 May, 1998)

\begin{abstract}
For each $m \geq 1, u_{m}(G)$ is defined to be the intersection of the normalizers of all the subnormal subgroups of defect at most $m$ in $G$. An ascending chain of subgroups $u_{m, i}(G)$ is defined by setting $u_{m, i}(G) / u_{m, i-1}(G)=u_{m}\left(G / u_{m, i-1}(G)\right)$. If $u_{m, n}(G)=G$, for some integer $n$, the $m$-Wielandt length of $G$ is the minimal of such $n$.

In [3], Bryce examined the structure of a finite soluble group with given $m$-Wielandt length, in terms of its polynilpotent structure. In this paper we extend his results to infinite soluble groups.
\end{abstract}

1991 Mathematics Subject Classification. 20E15, 20F22.

1. Introduction and main results. The Wielandt subgroup $w(G)$ of a group $G$ is defined to be the intersection of all normalizers of subnormal subgroups of $G$. It was introduced in 1958 by Wielandt [12], who showed that $w(G)$ contains every simple non-abelian subnormal subgroup, and every minimal normal subgroup which satisfies the minimal condition on subnormal subgroups. Thus $w(G)$ is non-trivial if $G$ is a finite non-trivial group. Conversely there exist infinite groups with trivial Wielandt subgroup; for example, the infinite dihedral group.

The Wielandt series of $G$ is defined as follows: write $w_{0}(G)=1$ and, for $i \geq 1$ define $w_{i}(G)$ inductively by the relation $w_{i}(G) / w_{i-1}(G)=w\left(G / w_{i-1}(G)\right)$. If for some integer $n, w_{n}(G)=G$, then $G$ is said to have finite Wielandt length, the Wielandt length of $G$ being the minimal $n$ such that $w_{n}(G)=G$. We denote by $\mathcal{W}_{n}$ the class of all groups whose Wielandt length is at most $n$. By the remark above, any finite group has finite Wielandt length, while in general this is not true for infinite groups.

The relation between the Wielandt length and the derived and Fitting length in a finite soluble group was first investigated by A. Camina in [4]; more recently R. Bryce and J. Cossey [2] improved on Camina's results by obtaining best possible bounds for both the derived and the Fitting length of a finite soluble group in terms of its Wielandt length. Finally C. Casolo in [5] showed that the same bounds hold for all soluble groups with finite Wielandt length.

In 1990, R. Bryce [3] introduced the subgroups $u_{m}(G)$, which are defined, for each $m \geq 1$, to be the intersection of the normalizers of all the subnormal subgroups of defect at most $m$ in $G$; he defined also new series, which we shall call $m$-Wielandt series, in the following way: for each $m \geq 1$ write $u_{m, 0}(G)=1$ and if $i \geq 1$ define $u_{m, i}(G)$ inductively by the relation $u_{m, i}(G) / u_{m, i-1}(G)=u_{m}\left(G / u_{m, i-1}(G)\right)$. If, for some integer $n, u_{m, n}(G)=G$, then $G$ is said to have finite $m$-Wielandt length, and the minimal such $n$ is called the $m$-Wielandt length of $G$. In his paper Bryce proved that there exist bounds on the derived and Fitting length of a finite soluble group in terms of its $m$-Wielandt length $(m \geq 2)$, which are analogous to those expressed in terms of the Wielandt length, and he determined the best possible bounds. Moreover he examined very closely the polynilpotent structure of a finite soluble group, in 
terms of its $m$-Wielandt length. Let $\mathcal{N}_{c}$ denote the class of nilpotent groups of nilpotency class at most $c$. Then, as usual, the product $\mathcal{N}_{c_{1}} \cdots \mathcal{N}_{c_{t}}$ denotes the class of groups that have a finite ascending series with nilpotent factors of nilpotency class respectively at most $c_{i},(i=1, \ldots, t)$. A group $G$ which belongs to $\mathcal{N}_{c_{1}} \cdots \mathcal{N}_{c_{t}}$ is said to have polynilpotent class row $\left(c_{t}, \ldots, c_{1}\right)$. (See Hanna Neumann $[\mathbf{8}$, p. 14].)

According to Bryce's definition, $\left(a_{1}, \ldots, a_{r}\right)$ is the minimal class row of $G$ if and only if the following three conditions are satisfied.

(i) $\left(a_{1}, \ldots, a_{r}\right)$ is a class row of $G$.

(ii) $r$ is the Fitting length of $G$.

(iii) $a_{i} \leq b_{i}$, for each $1 \leq i \leq r$, for every class row $\left(b_{1}, \ldots, b_{r}\right)$ of $G$.

A class of groups $\mathcal{X}$ has minimal class row $c=\left(a_{1}, \ldots, a_{r}\right)$ if all groups in $\mathcal{X}$ have class row $c$ and, whenever all groups in $\mathcal{X}$ have class row $\left(a_{1}^{\prime}, \ldots, a_{s}^{\prime}\right)$, then either $r<s$ or $s=r$ and $a_{i}^{\prime} \geq a_{i},(i=1, \ldots, r)$.

For $m \neq 3$ Bryce determined the minimal class row of the class of finite soluble groups with $m$-Wielandt length at most $n$. For $m=3$, he proved that the class of finite soluble groups with 3 -Wielandt length at most $n$ has a minimal class row, but if $n \geq 3$ he was not able to decide which it is between two possibilities.

In this paper we extend Bryce's results to infinite soluble groups and we determine the minimal class row of the class of soluble groups with 3-Wielandt length at most $n$, even in the case $n \geq 3$. Extending Bryce's notation, we denote by $\mathcal{W}_{m, n},(m \geq 2, n \geq 1)$, the class of all groups whose $m$-Wielandt length is at most $n ; \mathcal{A}$ denotes the class of all abelian groups, $\mathcal{A}^{2}$ the class of abelian groups of exponent at most 2. Thus $\mathcal{A}^{r}$ denotes the class of soluble groups of derived length at most $r$. Notation used for operations between classes of groups is consistent with [10].

Our main results are as follows.

Theorem 1. If $G$ is a soluble group in $\mathcal{W}_{2, n},(n \geq 2)$, then $G \in \mathcal{N}_{2} \mathcal{N}_{3}^{n-2} \mathcal{N}_{2} \mathcal{A}$.

THEOREM 2. If $G$ is a soluble group in $\mathcal{W}_{m, n},(m \geq 3, n \geq 1)$, then for $n \neq 2$ $G \in \mathcal{A N}_{2}^{n-1} \mathcal{A}$, while $G \in \mathcal{N}_{2}^{2} \mathcal{A}$ when $n=2$.

THEOREM 3. If $G$ is a soluble group in $\mathcal{W}_{2, n},(n \geq 2)$, then $G \in \mathcal{A}^{2 n+1}$. If $n=1$, then $G \in \mathcal{A}^{2}$.

THEOREM 4. If $G$ is a soluble group in $\mathcal{W}_{m, n},(m \geq 3, n \geq 1)$, then

(i) $G \in \mathcal{A}^{5 n / 3} \quad$ if $n \equiv 0(\bmod 3)$,

(ii) $G \in \mathcal{A}^{5(n-1) / 3+2} \quad$ if $n \equiv 1(\bmod 3)$,

(iii) $G \in \mathcal{A}^{5(n-2) / 3+3} \mathcal{A}_{2} \quad$ if $n \equiv 2(\bmod 3)$.

Clearly Theorems 1 and 2 say that for $n \geq 2$ the class $\mathcal{W}_{2, n}$ has class row $(1,2, \underbrace{3, \ldots, 3}_{n-2}, 2)$, while for $m \geq 3$ the class $\mathcal{W}_{m, n}$ has class row $(1, \underbrace{2, \ldots, 2}_{n-1}, 1)$ when $n \neq 2$ and $(1,2,2)$ when $n=2$. By Bryce's results, these are the minimal class rows for the considered classes.

Finally we prove that in order to conclude that a soluble 2-group is a Hamiltonian group it is enough to assume that $u_{4}(G)$ is Hamiltonian. A weaker result is already known for finite 2-groups. Such a group $G$ is Hamiltonian if $w(G)$ is Hamiltonian. This is a special case of the result of Baer [1, p. 246]. 
2. Preliminaries. We begin by listing some general properties of the $m$-Wielandt series that have been proved by Bryce in [3].

LEMMA 2.1. Let $G$ be a group.

(i) For $m \geq 1, i \geq 1$ the subgroups $u_{m, i}(G)$ are characteristic in $G$, $w_{i}(G) \leq u_{m, i}(G) \leq u_{m-1, i}(G) \leq \ldots \leq u_{1, i}(G)=G$ and

$$
w_{i}(G)=\bigcap_{m=1}^{\infty} u_{m, i}(G)
$$

Moreover $\mathcal{W}_{n}=\bigcap_{m=1}^{\infty} \mathcal{W}_{m, n}$.

(ii) If $N$ is a normal subgroup of $G$, for $m \geq 2$ and $i \geq 1$ we have $u_{m, i}(G) N / N \leq u_{m, i}(G / N)$ and $u_{m, i}(G) \cap N \leq u_{m-1, i}(N)$.

(iii) For $m \geq 2, n \geq 1$ the class $\mathcal{W}_{m, n}$ is closed for quotient groups and $\mathcal{W}_{m, n} \subseteq \mathcal{W}_{m-1, n}$

(iv) For $m \geq 2$ and $1 \leq i \leq m-1$ every group in $\mathcal{W}_{m, i}$ has the property that its subnormal subgroups have defect at most $i$.

A group is said to be a T-group if every subnormal subgroup is normal. The structure of soluble T-groups has been studied by Zacher [13], Gaschütz [7] and Robinson [9]; in particular, it is known that the Fitting subgroup of a soluble Tgroup (which coincides with the centralizer of the derived subgroup) is a Dedekind group, and a soluble T-group is metabelian.

Following Bryce's notation in [3], we denote by $\mathcal{J}$ the class defined as follows: $G \in \mathcal{J}$ if and only if, whenever $C$ and $D$ are characteristic subgroups of $G$ such that $D \leq C$ and $C / D$ is abelian, then every subgroup of $G$ that lies between $C$ and $D$ is normal in $G$. It is clear that T-groups belong to $\mathcal{J}$, and that $\mathcal{J}$ is closed for characteristic subgroups and factor groups by characteristic subgroups.

Lemma 2.2 ([3, Lemmas 3.6, 3.7]). Let $G$ be a group. For $m \geq 3$ the subgroup $u_{m}(G)$ is a T-group, while $u_{2}(G) \in \mathcal{J}$.

Recall that if $A$ is a group, a power automorphism of $A$ is an automorphism mapping every subgroup of $A$ onto itself. Cooper in [6] proved that the group of power automorphisms, $P$ Aut $A$, is an abelian normal subgroup of Aut $A$, and if $A$ is abelian, $P$ Aut $A \leq Z$ (Aut $A)$.

Finally if $G$ is a group, we denote by $F(G)$ the Fitting subgroup of $G$.

3. Proof of Theorems 2 and 4. Lemma 2.2 implies that for $m \geq 3, n \geq 1$, if a group belongs to $\mathcal{W}_{m, n}$, then it has a characteristic series whose factors are T-groups. Thus Theorems 2 and 4 are particular cases of two more general results valid for soluble groups with a normal series whose factors are T-groups.

The following proposition may be compared to Lemma 4 in [5].

Proposition 3.1. Let $G$ be a soluble group and let $Z$ be a central subgroup of $G$ such that $G / Z$ is a $T$-group. Then the Fitting subgroup $F$ of $G$ is nilpotent of class at most 2 and $[G, \operatorname{Aut} G] \leq F$. 
Proof. It is clear that we may assume that $Z$ is the centre of $G$ and hence is a characteristic subgroup of $G$. We show first that $F$ is nilpotent of class at most 2 . Note that $F / Z$ is actually the Fitting subgroup of $G / Z$; then $G^{\prime} \leq F$ and $F / Z$ is a Dedekind group, since $G / Z$ is a soluble T-group. If $F / Z$ is abelian, we get immediately that $F$ is nilpotent of class at most 2 . If $F / Z$ is not abelian, then it is periodic and it can be written as $F / Z=Q / Z \times A / Z \times B / Z$, where $Q / Z$ is the quaternion group of order $8, A / Z$ is an elementary abelian 2-group and $B / Z$ is an abelian group with all the elements of odd order. Then $Q=\langle x, y, Z\rangle$ where $y^{4} \in Z$, $x^{2} \equiv y^{2}(\bmod Z)$ and $x^{y} x \in Z$. If $D / Z=(F / Z)^{\prime}$, then $D=\left\langle y^{2}, Z\right\rangle$ and $y^{2}$ centralizes $F$ modulo $Z$. We show that actually $D$ lies in the centre of $F$.

Let $b \in B \backslash Z$. Since every element of $B / Z$ has odd order, there exists an odd integer $m$ such that $b^{m} \in Z$. Thus, since $\left[y^{2}, b\right] \in Z$, we have that

$$
1=\left[y^{2}, b^{m}\right]=\left[y^{2}, b\right]^{m} \text {. }
$$

On the other hand

$$
1=\left[y^{4}, b\right]=\left[y^{2}, b\right]^{2}
$$

and so, since $(m, 2)=1,\left[y^{2}, b\right]=1$. If $a \in A \backslash Z$, then $a^{2} \in Z$ and $[y, a] \in Z$, whence

$$
1=\left[y, a^{2}\right]=[y, a]^{2}=\left[y^{2}, a\right] .
$$

Finally since $y^{2}=x^{2} z$ for some $z \in Z$, we have

$$
\left[x, y^{2}\right]=\left[x, x^{2} z\right]=\left[x, x^{2}\right]=1 .
$$

Hence $y^{2} \in Z(F)$, so that $D \leq Z(F)$, and $F$ is nilpotent of class 2 .

Now set $C=C_{G}(F / D)$; then $C$ acts trivially on the series $G \geq F \geq D \geq Z \geq 1$ (because $G / F$ and $Z$ are central in $G$ and $D / Z$ has order 2), so that $C$ is a normal nilpotent subgroup of $G$ and hence $C \leq F$. On the other hand $F / D$ is abelian and it a characteristic factor of $G$; hence $G$ acts by conjugation as a group of power automorphisms on $F / D$ and so $[G, \operatorname{Aut} G] \leq C \leq F$, since power automorphisms of an abelian group commute with every automorphism.

COROllary 3.2. Let $G$ be a soluble group, let $W \triangleleft G$ be a T-group, and let $N=F(W)$. Then $W / N \leq Z(G / N)$.

Proof. By Proposition 3.1, [W, Aut $W] \leq N$. Since $W$ is normal in $G, G$ acts on $W$ as a group of automorphisms and so $[W, G] \leq[W$, Aut $W] \leq N$.

Notice that the same result has been proved by Casolo [5, Lemma 2] when $W=w(G)$.

If $G$ is a group, for every prime number $p$ we denote by $O_{p^{\prime}}(G)$ the subgroup of $G$ generated by the $p^{\prime}$-normal subgroups of $G$.

THEOREM 3.3. Let $G$ be a soluble group with a normal series of length $n \geq 1$ whose factors are T-groups. Then $G \in \mathcal{A N}_{2}^{n-1} \mathcal{A}$, if $n \neq 2$, while if $n=2$, then $G \in \mathcal{N}_{2}^{2} \mathcal{A}$.

Proof. Let $1=G_{0} \leq G_{1} \leq \ldots \leq G_{n}=G$ be the series of $G$. First of all we show that in each case $G \in \mathcal{N}_{2}^{n} \mathcal{A}$. For each $i=1, \ldots, n$ define the subgroups $N_{i}$ by means of the relation $N_{i} / G_{i-1}=F\left(G_{i} / G_{i-1}\right)$. Then for each $i, G_{i} / G_{i-1}$ is a soluble T-group 
and so, by Corollary 3.2, $G_{i} / N_{i}$ is central in $G$. Moreover $N_{1}$ is a Dedekind group and so it is nilpotent of class at most 2; $N_{i+1} / N_{i}$ is nilpotent of class at most 2 for each $i=1, \ldots, n-1$, by Proposition 3.1, and $G / N_{n}$ is abelian because $[G$, Aut $G] \leq N_{n}$. Hence $G \in \mathcal{N}_{2}^{n} \mathcal{A}$ as required.

Now if $n=2$ we are done. If $n=1, G$ is a soluble T-group and so $G \in \mathcal{A}^{2}$. Let us suppose that $n \geq 3$. Since the class $\mathcal{A N}_{2}^{n-1} \mathcal{A}$ is residually closed it is enough to prove the theorem when $O_{p^{\prime}}(G)=1$, for an arbitrary prime $p$. First we show that $G_{3}$ contains a normal subgroup $D \in \mathcal{A N}_{2}^{2}$ such that $\left[G_{3}, G\right] \leq D$. Since $O_{p^{\prime}}(G)=1$ then either $N_{1}$ is not periodic or $N_{1}$ is a $p$-group. If $N_{1}$ is abelian, by the first part of the proof we can choose $D=N_{3}$. Then suppose that $N_{1}$ is non-abelian; in such a case $p=2$ and this implies (see Robinson [9]) that $G_{1}=N_{1}$ is a non-abelian Dedekind 2group. If $X=Z\left(G_{1}\right)$, then $X$ is a normal subgroup of $G$ and $G_{1} / X$ is the elementary abelian group of order 4 .

Set $C=C_{G_{3}}\left(G_{1} / X\right)$; then $G_{3} / C$ is isomorphic to a subgroup of $\operatorname{Aut}\left(G_{1} / X\right) \simeq S_{3}$. Let $L / C$ be the inverse image of $A_{3}$ with respect to the embedding of $G_{3} / C$ in $S_{3}$ and set $D=L \cap N_{3}$. In order to show that $\left[G_{3}, G\right] \leq D$, observe that $L$ is a normal subgroup of $G$ of index at most two in $G_{3}$ and so $\left[G_{3}, G\right] \leq L$. On the other hand $\left[G_{3}, G\right] \leq N_{3}$; thus $\left[G_{3}, G\right] \leq D$. It remains to show that $D \in \mathcal{A N}_{2}^{2}$. First consider $D /\left(C \cap N_{2}\right)$. Since $N_{3} / N_{2}$ is nilpotent of class at most 2, its derived subgroup, say $R / N_{2}$, is a central factor in $N_{3}$ and so $[D, C \cap R] \leq C \cap N_{2}$; that is to say $(C \cap R) /\left(C \cap N_{2}\right) \leq Z\left(D /\left(C \cap N_{2}\right)\right)$; on the other hand $D^{\prime} \leq L^{\prime} \cap N_{3}^{\prime} \leq C \cap R$. Hence $D /\left(C \cap N_{2}\right)$ is nilpotent of class at most 2. Now $\left(C \cap N_{2}\right) / G_{1}$ is a nilpotent T-group and $G_{1} / X$ is central in $\left(C \cap N_{2}\right) / X$. Thus, by Proposition 3.1, we get that $\left(C \cap N_{2}\right) / X$ is nilpotent of class at most 2 , and $D \in \mathcal{A N}_{2}^{2}$.

Now if $n=3$ we are done. If $n>3$, consider the series $D \leq G_{3} \leq$ $N_{4} \leq \ldots \leq N_{n} \leq G$, where $N_{i}$ are defined as above. Then, by Proposition 3.1, $N_{4} / D$ is nilpotent of class at most two. Hence $G / D \in \mathcal{N}_{2}^{n-3} \mathcal{A}$ and $G \in \mathcal{A N}_{2}^{n-1} \mathcal{A}$.

The following theorem extends the results of Theorem 2 in [5] and uses almost the same proof given by Casolo. (See [5, p. 333].)

THEOREM 3.4. Let $G$ be a soluble group with a normal series of length $n \geq 1$, whose factors are T-groups. Then

(i) if $n \equiv 0(\bmod 3)$ we have $G \in \mathcal{A}^{5 n / 3}$,

(ii) if $n \equiv 1(\bmod 3)$ we have $G \in \mathcal{A}^{5(n-1) / 3+2}$,

(iii) if $n \equiv 2(\bmod 3)$ we have $G \in \mathcal{A}^{5(n-2) / 3+3} \mathcal{A}_{2}$.

Proof. Let $1=G_{0} \leq G_{1} \leq \ldots \leq G_{n}=G$ be the series of $G$, and proceed by induction on $n$. If $n=1$ the result follows from Theorem 3.3. If $n=2,3$, notice that the proof of Lemma 5 in [5] works if we put $G_{1}$ and $G_{2}$ in the place of $w(G)$ and $w_{2}(G)$ respectively. Let $n>3$, and let the result be true for all groups with a normal series of length less than $n$. Then $G / G_{3}$ satisfies the inductive hypothesis and so we deduce the following results:

(i) if $n \equiv 0(\bmod 3)$, then $G / G_{3} \in \mathcal{A}^{5(n-3) / 3}, G_{3} \in \mathcal{A}^{5}$, whence $G \in \mathcal{A}^{5 n / 3}$,

(ii) if $n \equiv 1(\bmod 3)$, then $G / G_{3} \in \mathcal{A}^{5(n-4) / 3+2}, G_{3} \in \mathcal{A}^{5}$, whence $G \in \mathcal{A}^{5(n-1) / 3+2}$,

(iii) if $n \equiv 0(\bmod 3)$, then $G / G_{3} \in \mathcal{A}^{5(n-5) / 3+3} \mathcal{A}_{2}, G_{3} \in \mathcal{A}^{5}$, whence $G \in \mathcal{A}^{5(n-2) / 3+3}$. 
Proof of Theorems 2 and 4. Consider the $m$-Wielandt series of $G$

$$
1 \leq u_{m}(G) \leq \ldots \leq u_{m, n-1}(G) \leq u_{m, n}(G)=G .
$$

By Lemma 2.2 each factor $u_{m, i+1}(G) / u_{m, i}(G)$ is a T-group. Thus, by applying Theorem 3.3 and Theorem 3.4 respectively, we can conclude the proof.

4. Proof of Theorems 1 and 3. A direct consequence of Lemma 2.2 is that groups in $\mathcal{W}_{2, n}$ have a characteristic series whose factors belong to $\mathcal{J}$. As in the previous paragraph we shall state Theorems 1 and 3 as corollaries of more general theorems which hold for soluble groups with a normal series whose factors lie in $\mathcal{J}$.

Lemma 4.1. Let $G$ be a soluble group in $\mathcal{J}$. Then $G$ has a characteristic nilpotent subgroup $C$, with class at most 2 , such that $[G, \operatorname{Aut} G] \leq C$.

Proof. Let $H / K$ be an abelian characteristic factor of $G$ : then $G$ acts by conjugation on $H / K$ as a group of power automorphisms, and thus $[G, \operatorname{Aut} G] \leq$ $C_{G}(H / K)$. Let $C$ be the intersection of the centralizers of all the abelian characteristic factors of $G$. Then obviously $C$ is a characteristic subgroup of $G$ and $[G, \operatorname{Aut} G] \leq C$. Moreover the derived series of $C$ is central in $C$, so that $C$ is nilpotent and

$$
\gamma_{3}(C)=\left[C, C^{\prime}\right] \leq C^{\prime \prime} \leq \gamma_{4}(C)
$$

thus $\gamma_{3}(C)=\gamma_{4}(C)=1$.

Theorem 4.2. Let $G$ be a soluble group, with a normal series $1=G_{0} \leq$ $G_{1} \leq \ldots \leq G_{n}=G$ of length $n \geq 1$, whose factors belong to $\mathcal{J}$. Then $G \in \mathcal{N}_{2} \mathcal{N}_{3}^{n-1} \mathcal{A}$, and has derived length at most $2 n+1$. If also $n \geq 2$ and $G / G_{n-1}$ is a T-group, then $G \in \mathcal{N}_{2} \mathcal{N}_{3}^{n-2} \mathcal{N}_{2} \mathcal{A}$.

Proof. By Lemma 4.1, for each $i=0, \ldots, n-1$, there exists a subgroup $C_{i}$ such that $C_{i} / G_{i}$ is a characteristic nilpotent subgroup of $G_{i+1} / G_{i}$ with class at most 2 , and $G_{i+1} / C_{i}$ is central in $G / C_{i}$. Then $C_{i+1} / C_{i}$ is nilpotent of class at most 3, for each $i=0, \ldots, n-2, C_{0}$ is nilpotent of class at most 2 and $G / C_{n-1}$ is abelian. Hence $G \in \mathcal{N}_{2} \mathcal{N}_{3}^{n-1} \mathcal{A}$. Moreover since a nilpotent group of class at most 3 is metabelian, it follows immediately that $G \in \mathcal{A}^{2 n+1}$. Suppose now that $n \geq 2$ and $G / G_{n-1}$ is a Tgroup; then, by Proposition 3.1, $C_{n-1} / C_{n-2}$ has class at most 2 . Hence $G \in \mathcal{N}_{2} \mathcal{N}_{3}^{n-2} \mathcal{N}_{2} \mathcal{A}$.

Proof of Theorems 1 and 3. Consider the 2-Wielandt series of $G$

$$
1 \leq u_{2}(G) \leq \ldots \leq u_{2, n-1}(G) \leq u_{2, n}(G)=G .
$$

Then for $i=0, \ldots, n-2$, by Lemma 2.2 each factor $u_{2, i+1}(G) / u_{2, i}(G)$ belongs to $\mathcal{J}$ and $G / u_{2, n-1}(G)$ is a T-group. Thus when $n \neq 1$ by Theorem 4.2 we get the conclusion. If $n=1$, then $G=u_{2}(G)$ is a soluble T-group by Lemma 2.1(iv) and it is metabelian.

The kern $K(G)$ of a group $G$ is defined as the subgroup of all elements that normalize every subgroup of $G$. Clearly $K(G)$ is a Dedekind group. Baer in [1] 
showed that if $G$ is a 2-group and $K(G)$ is not abelian, then $G=K(G)$ or equivalently $G$ is Hamiltonian. Since finite $p$-groups are nilpotent, if $G$ is a finite 2-group $w(G)=K(G)$, and so $w(G)$ is not abelian if and only if $G$ is Hamiltonian. Bryce generalized this result proving that if $G$ is a finite 2-group such that $u_{3}(G)$ is not abelian, then $G$ is Hamiltonian. Here we investigate what happens for infinite soluble 2-groups.

Theorem 4.3. Let $G$ be a soluble 2-group and let $u_{4}(G)$ be Hamiltonian. Then $G$ is Hamiltonian.

In order to prove this result we need the following lemma.

Lemma 4.4 ([3, Lemma 5.5]). Let $H$ be a 2-group containing a Hamiltonian subgroup $K$ of index 2. If $K \cap u_{2}(H)$ is non-abelian, then $H$ itself is a Hamiltonian group.

Note that although Bryce's article [3], according to his introduction is about finite groups, the proof of Lemma 5.5 works also for infinite groups, and so it actually proves our Lemma 4.4.

Proof of Theorem 4.3. Let $1=G_{0} \leq u_{4}(G)=G_{1} \leq G_{2} \leq \ldots \leq G_{n}=G$ be an abelian normal series of $G$; we prove by induction on $i$ that $G_{i}$ is Hamiltonian, $(i=1, \ldots, n)$. If $i=1, G_{1}=u_{4}(G)$ and there is nothing to prove. If $i>1$, assume that $G_{i-1}$ is Hamiltonian and let us prove that $G_{i}$ is Hamiltonian. Let $S \geq G_{i-1}$ be a subgroup of $G_{i}$ such that $S / G_{i-1}$ is finite and let $G_{i-1}=H_{0} \leq H_{1} \leq \ldots \leq H_{t}=S$ be part of a chief series of it. Then by Lemma 2.1(ii), $u_{4}(G)=u_{4}(G) \cap G_{i} \cap H_{j} \leq$ $u_{3}\left(G_{i}\right) \cap H_{j} \leq u_{2}\left(H_{j}\right)$ and so Lemma 4.4 yields that $H_{j}$ is Hamiltonian provided that $H_{j-1}$ is. Thus, by induction, $S$ is Hamiltonian; then $G_{i}$ is locally Dedekindian and so Hamiltonian. This completes the initial induction and we can conclude that $G=G_{n}$ is Hamiltonian.

Note that in contrast to what happens for finite groups, it is not sufficient to assume that $u_{4}(G)$ is non-abelian, since there exist infinite soluble 2-groups that are Tgroups, but they are neither abelian nor Hamiltonian; (see Robinson [9]). By Robinson [9], the condition " $u_{4}(G)$ Hamiltonian" is equivalent to " $F\left(u_{4}(G)\right)$ non-abelian".

One can see that if $G$ is a nilpotent 2-group, then $u_{4}(G)$ in Theorem 4.3 can be replaced by $u_{3}(G)$; we don't know if this fact holds when $G$ is any soluble 2-group.

\section{REFERENCES}

1. R. Baer, Gruppen mit hamiltonshem Kern, Compositio Math. 2 (1935), 241-246.

2. R. A. Bryce and J. Cossey, The Wielandt subgroup of a finite soluble group, J. London Math. Soc. (2) 40 (1989), 244-256.

3. R. A. Bryce, Subgroups like Wielandt's in finite soluble groups, Math. Proc. Cambridge Phil. Soc. 107 (1990), 239-259.

4. A. R. Camina, The Wielandt length of finite groups, J. Algebra 15 (1970), 142-148.

5. C. Casolo, Soluble groups with finite Wielandt length, Glasgow Math. J. 31 (1989), $329-334$.

6. C. D. H. Cooper, Power automorphisms of a group, Math. Z. 107 (1968), 335-356.

7. W. Gaschütz, Gruppen in denen das Normalteilersein transitiv ist, J. Reine Angew. Math. 198 (1957), 87-92. 
8. H. Neumann, Varieties of groups (Springer-Verlag, 1967).

9. D. J. S. Robinson, Groups in which normality is a transitive relation, Proc. Cambridge Phil. Soc. 60 (1964), 21-38.

10. D. J. S. Robinson, Finitness conditions and generalized soluble groups, Part 1 (Springer-Verlag, 1972).

11. D. J. S. Robinson, A course in the theory of groups (Springer-Verlag, 1982).

12. H. Wielandt, Uber den Normalisator der subnormalen Untergruppen, Math. Z. 69 (1958), 463-465.

13. G. Zacher, Caratterizzazione dei t-gruppi finiti risolubili, Ricerche Mat. 1 (1952), 287-294. 